\title{
Gold Nanoparticle Treated Textile-Based Materials for Potential use as Wearable Sensors
}

\author{
Kam Ling Chan¹, Derek Fawcett', Gérrard Eddy Jai Poinern¹
}

Murdoch Applied Nanotechnology Research Group. Department of Physics, Energy Studies and Nanotechnology, School of Engineering and Energy, Murdoch University, Murdoch, Western Australia 6150, Australia.

\begin{abstract}
Wearable sensors for monitoring and clinical applications for non-hospital-based healthcare has the potential to significantly reduce healthcare costs and permit individuals to maintain an independent lifestyle free of the constraints imposed by hospital-based care. Wearable, small chemical sensors have the potential to deliver a wide range of reliable on body monitoring systems for personal monitoring and detecting chemicals in the environment. The present work investigates the potential of using widely available commercial fabric materials as chemical sensors. Various fabrics including natural, synthetics and blends of both natural and synthetic were treated with gold nanoparticle solutions to determine the treatments effectiveness in changing a materials electrical resistance. The studies found treating the fabrics reduced the materials resistive characteristics. The study also found that treated natural silk fabrics could also be used to detect ethanol vapour. Thus, making treated silk fabrics a potential chemical sensor.
\end{abstract}

Keywords: textiles, gold nanoparticles, electrical resistance, wearable, chemical sensors

\section{Introduction}

Today chemical sensors are widely used either singly to detect a specific chemical or collectively in arrays to sense or analyse complex mixtures of chemicals. A wide variety of materials including polymers, ferroelectric ceramics, piezoelectric semiconductors and shape memory films have been studied and used to manufacture an assortment of chemical sensors [13 ]. These sensors have been used in a wide variety of applications such as gas detection, food processing, cosmetics and pharmaceuticals [4-8]. In recent years, textiles have also been considered as a possible avenue for developing personal sensing and monitoring systems for medical, health \& safety and military applications [9-11]. Historically, various forms of textile materials have been universally worn by humanity for thousands of years. Accordingly, over time, changing requirements and fashions, individuals have readily changed their clothing styles to meet the challenges of the day. Thus, textiles are easily available, widely used and offer a unique platform that can be incorporated into the design of wearable sensors and personal monitoring systems. Currently, wearable sensors have been used in a variety of healthcare monitoring and diagnostic applications. In particular, physiological monitoring can significantly contribute to diagnosis, promote ongoing treatment and assist in patient rehabilitation

\begin{abstract}
[12]. These wearable sensors provide continuous monitoring and real-time information that allows individuals the opportunity to maintain a healthy lifestyle. Furthermore, present wearable sensors can detect motion and undertake physiological and biochemical sensing, which is particularly important when monitoring drug treatment efficacy [13, 14]. Recent research into healthcare delivery reflects a growing interest in home-based patient care as opposed to more conventional centralized hospitalbased care. Home-based patient management via wearable sensors has the potential to significantly reduce health-care costs and allows patients to maintain their independent lifestyle free of the constraints imposed by hospital-based care.
\end{abstract}

The PC industry has seen great strides in miniaturisation of various electronic components. Microelectronic-based manufacturing technologies integrated with textiles with advantageous electrical properties are important factors that can contribute to the successful development of wearable sensor systems. Modern high performance textile fabrics have been developed for a variety of sportswear that promote moisture repelling and temperature regulating properties [15]. And recent studies have also shown that wearable sweat-based electrochemical biosensors can monitor sodium,

This article is published under the terms of the Creative Commons Attribution License 4.0

Author(s) retain the copyright of this article. Publication rights with Alkhaer Publications.

Published at: http://www.ijsciences.com/pub/issue/2016-05/

DOI: 10.18483/ijSci.1018; Online ISSN: 2305-3925; Print ISSN: 2410-4477 
chloride and lactate levels [16, 17]. Accordingly, developing electrically enhanced textile fabrics will be at the forefront of smart clothing with the ability to sense and communicate information to remote data collection centres via the internet [18].

Textiles are planar fibrous materials with unique rheological properties that allow them to behave differently when compared to more conventional engineering materials. The rheological properties of textiles include elasticity, viscosity, plasticity and strength. The individual component fibres and the arrangement of the yarn or fabric largely determine the properties. The orientation of fibres and yarns introduces an intrinsic planar anisotropy and nonhomogeneous nature to the properties of textiles [19]. The orientation of fibres and yarns also creates a porous structure, in which fibre density and pore volume directly influences properties such as air permeability and thermal insulation. Also the flexible and compressible nature of the porous structure can also accommodate changes in thickness and density when the textile is subjected to pressure. Another interesting feature of textiles that influences their morphology and material properties results from the continuous exchange of water molecules with the surrounding environment. Furthermore, the electrical resistance of textiles depends directly on the individual fibres and the geometry the woven structure forming the fabric. Interestingly, textile fibres are considered insulating materials with typical electrical resistances in the order of $10^{12} \Omega$ [20]. The present paper deals with measuring the electrical resistance of several different types of textiles that have been treated with gold $(\mathrm{Au})$ nanoparticle solutions. Textiles examined included natural fibres (cotton, silk and wool), synthetic fibres (polyester, nylon), semi-synthetic (viscose) and a number of common blends of natural and synthetic fibres.

\section{Materials and methods}

\subsection{Materials}

The source of $\mathrm{Au}^{+}$ions were derived from chloroauric acid $\mathrm{HAuCl}_{4}$ (purity: 99.99\%), the reducing agent used was sodium borohydride, $\left(\mathrm{NaBH}_{4}\right)$ and the capping agent used was sodium citrate $\left(\mathrm{C}_{6} \mathrm{H}_{5} \mathrm{Na}_{3} \mathrm{O}_{7}\right)$. All chemicals were supplied by Sigma-Aldrich (Castle Hill, NSW Australia) and used without any further purification. All aqueous solutions were prepared using Milli- $Q^{\circledR}$ water produced by a Barnstead Ultrapure Water System D11931 (Thermo Scientific Dubuque IA 18.3 M $\Omega$ $\left.\mathrm{cm}^{-1}\right)$.

\subsection{Preparation of Au nanoparticle solution}

Nanoparticles were produced by first adding a 3.0 $\mathrm{mL}$ solution of $0.001 \mathrm{M} \mathrm{HAuCl}_{4}$ to a $3.0 \mathrm{ml}$ solution of $0.001 \mathrm{M}$ sodium citrate (stabilising and capping agent) at room temperature $\left(24{ }^{\circ} \mathrm{C}\right)$ and agitating for 10 minutes. Reduction of $\mathrm{AuCl}_{4}^{-}$ions was initiated by the addition of a $3.0 \mathrm{~mL}$ solution of $0.1 \mathrm{M}$ sodium borohydride to the mixture. The reaction process was allowed to proceed at room temperature. Nanoparticle formation was confirmed by the reaction mixture changing to a dark purple colour and the use of a small laser pointer to reveal Tyndall scattering behaviour normally associated with nanoparticles in suspension.

\subsection{Types of Fabrics examined}

Several natural, synthetic and blended fabrics were examined using scanning electron microscopy to determine fabric properties such as fibre size and shape and fabric structure. Table 1 presents a listing of the various fabrics examined and their respective designations used to identify them in the voltagecurrent studies.

Table 1. Fabric materials examined and their respective designations in the present study

\begin{tabular}{lll}
\hline Material type & Material & Sample designations \\
\hline Natural & Cotton & Cotton \\
& Silk & Silk \\
& Wool & Wool \\
Synthetic & Polyester & Polyester \\
& Nylon & Nylon \\
Blends & $60 \%$ Cotton \& 40\% polyester & $6 \mathrm{C} 4 \mathrm{P}$ \\
& $60 \%$ Wool \& 40\% polyester & $6 \mathrm{~W} 4 \mathrm{P}$ \\
& $50 \%$ Wool \& 50\% viscose & $5 \mathrm{~W} 5 \mathrm{~V}$ \\
& $10 \%$ Wool \& 90\% viscose & $1 \mathrm{~W} 9 \mathrm{~V}$ \\
\hline
\end{tabular}

\subsection{Fabric treatments}

The first treatment procedure was to place droplets of the $\mathrm{Au}$ nanoparticle solution directly onto fabric samples to investigate the absorption behaviour of each material. Each fabric type was mounted onto a glass slide and then via drop-wise deposition, the $\mathrm{Au}$ nanoparticle solution was applied to the samples. The absorption behaviour of each fabric was observed and 
recorded. The second treatment involved immersing fabric samples in the $\mathrm{Au}$ nanoparticle solution for 20 and $73 \mathrm{~h}$ periods. After the respective immersion period, the samples were removed from the solution and allowed to air dry at room temperature $\left(24^{\circ} \mathrm{C}\right)$.

\subsection{Resistance measurements}

Electrical resistance measurements of both untreated and treated fabric samples were carried out using a two probe method. Sample testing was carried out at in an air-conditioned laboratory at room temperature $\left(24{ }^{\circ} \mathrm{C}\right.$ and relative humidity $\left.\sim 70 \%\right)$. Resistance measurements were taken at different locations on the respective samples with the sample distance between probes $(\sim 3 \mathrm{~mm})$. Measurements were carried out in triplicate using a Keithley 480 Picoammeter and standard DC laboratory power supply. The resulting voltage-current measurements were used to plot electrical characteristics of the treated fabrics and determine the changes in resistance of the fabrics.

The second set of resistance measurements investigated the ability of a treated silk fabric to detect the presence of ethanol vapour. A similar experimental procedure was followed as discussed above, the only difference being the silk fabric sample was mounted on two electrical contacts inside a small glass chamber. A small amount of ethanol was placed in the bottom of the test chamber, once sealed the pressure was dropped to produce a vaporous atmosphere inside the chamber. Voltage and current measurements were carried out and the resulting data was plotted and analysed to determine the electrical characteristics of the treated fabric and its response to ethanol vapours.

\subsection{Scanning electron microscopy studies}

A Cressington 208HR High Resolution Sputter coater was used to sputter coat samples with a $2 \mathrm{~nm}$ layer of gold to prevent charge build prior to Scanning electron microscope (SEM) examination. The fabric samples were mounted on standard SEM aluminium stubs. SEM micrographs were taken using a JCM6000 (Neo-Scope TM) to determine fibre size, morphology and woven structure of the respective fabric samples.

\section{Results and Discussions}

All fabric samples were examined using scanning electron microscopy to determine fibre sizes and the orientation of the fibres in the respective yarns. This examination was important, since it's the orientation of the fibres that creates the unique porous structure. Each sample displayed different porous structures and accounts for each fabric having different fabric properties such as air permeability and thermal insulation. Figure 1 presents a selection of low magnification images of the various fabrics examined in the present study. Figure 2 presents enlarged images for two natural fibres (Wool \& Cotton) and two common blends that combine natural fibres with synthetics. Figure 2(a) presents an image of a pure wool sample and Figure 2(b) shows an image of a common wool-viscose 50/50 blend (5W5V). Another commonly used natural fibre is cotton and an enlarged image of a cotton sample is presented in Figure 2(c). While a commonly produced blend containing cotton and synthetic polyester $(6 \mathrm{C} 4 \mathrm{P})$ is presented in Figure 2(d). Inspection of the pure wool sample in Figure 2(a) reveals that there is a certain degree of order in the fibre arrays. The loosely packed cylindrical fibres range in size from 20.9 to $24.1 \mu \mathrm{m}$ in diameter and also exhibit a large degree of spacing between individual fibres. The fibres also display a certain degree of curvature along their long lengths. The wool-viscose blend $(5 \mathrm{~W} 5 \mathrm{~V})$ presented in Figure 2(b) reveals the viscose fibres are cylindrical and range in diameter from 11.9 up to around $14.8 \mu \mathrm{m}$. The individual fibres have a smooth surface and are composed of multiple thinner fibres. The image also reveals a certain degree of order within the fabric and the presence of some loosely packed fibres. A pure cotton sample is presented in Figure 2(c) and reveals long, flatten and twisted fibres. The fibres are between 16.4 and $22.2 \mu \mathrm{m}$ in diameter and show a definite helix geometry over their entire length. The fibres are closely packed with some degree of spacing between individual fibres and some degree of loose packing in the overall fabric structure. A cotton \& polyester blend $(6 \mathrm{C} 4 \mathrm{P})$ is presented in Figure 2(d) and shows no fixed order within the fabric, which makes it quite unique when compared to the other fabric samples presented in Figure 1. The disorder in the 6C4P fabric can easily be seen in both Figure 1(f) and the enlarged image presented in Figure 2(d). Both images confirm the extent of disorder in the sample. Individual polyester fibres are smooth, long and have diameters that are around $10 \mu \mathrm{m}$. 


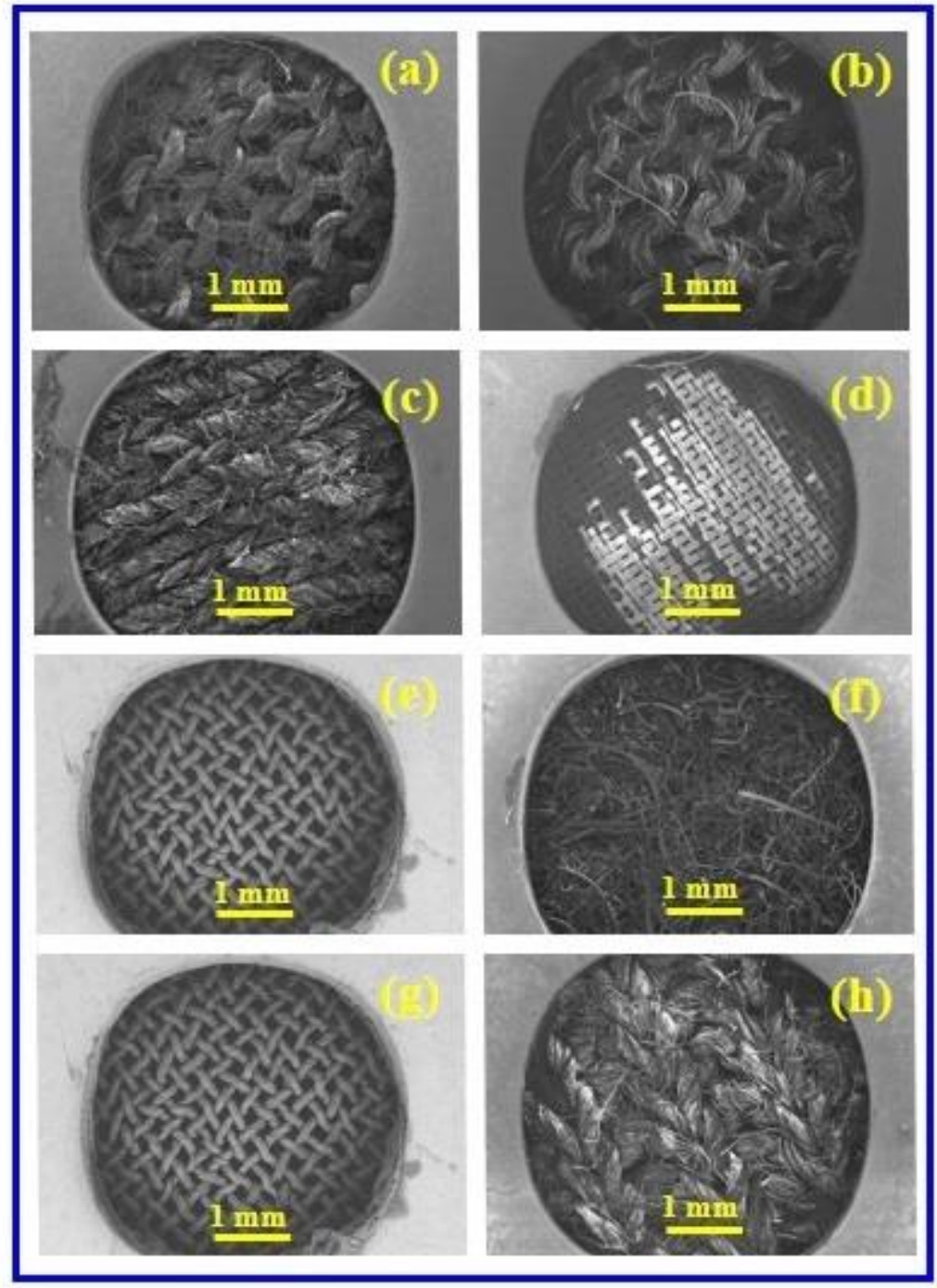

Figure 1. A selection of low magnification SEM images of the various fabrics: (a) Silk; (b) Wool; (c) Cotton; (d) Nylon; (e) Polyester; (f) 6C4P, (g) 6W4P, and (h) 1W9V

Figure 3 presents two enlarged images for two synthetic fibres (Polyester \& Nylon) and two enlarged images of natural silk. The polyester sample presented in Figure 3(a) shows a highly ordered and tightly packed woven fabric. The individual cylindrical smooth fibres range in diameter from 10.1 to $11.2 \mu \mathrm{m}$ and display a small degree of twisting within the arrangement that results in a small amount of spacing between individual fibres. A representative nylon sample is presented in Figure 3(b) and reveals semi-flattened cylindrical fibres that are smooth and tightly packed within a highly ordered woven fabric. The fibre diameters range between 14.4 and $15.6 \mu \mathrm{m}$ and display a consistent size distribution throughout the sample. Nylon was the only fabric that displayed a consistent closely packed and regular pattern within the fabric. The nylon sample also showed very little spacing between individual fibres. The remaining natural fibre investigated in the present work was silk and two enlarged images are presented in Figures 3(c) and (d). Inspection of each image reveals that silk fibres are cylindrical in cross-section and have diameters between 10.2 and $11.9 \mu \mathrm{m}$. The fibres are smooth, long and tend to have natural curvature without individual fibre twisting. Another feature of the fibres can also be seen in the images and involves fibres attaching to neighbour fibres in groups of 2 and 3 to form a flattened fibre profile. Silk also displays some degree of order, but there is also some loose packing seen throughout the fabric. 


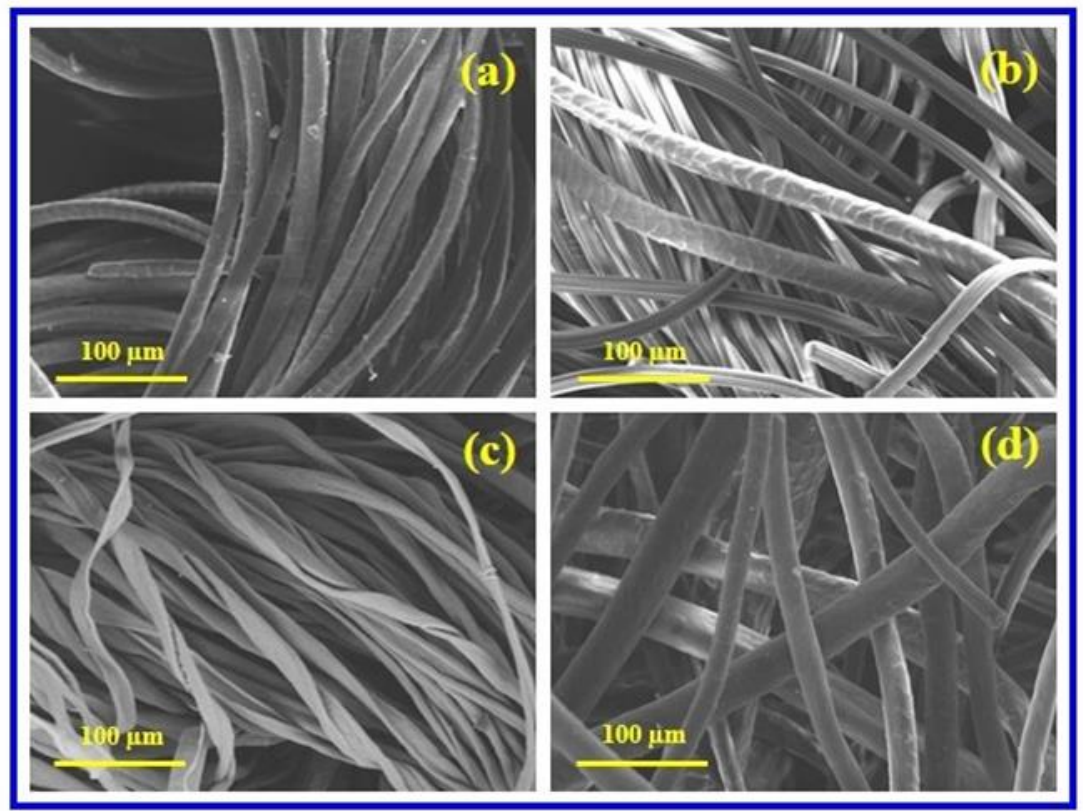

Figure 2. Enlarged images of fabric fibres: (a) Wool; (b) 5W5V; (c) Cotton, and (d) 6C4P

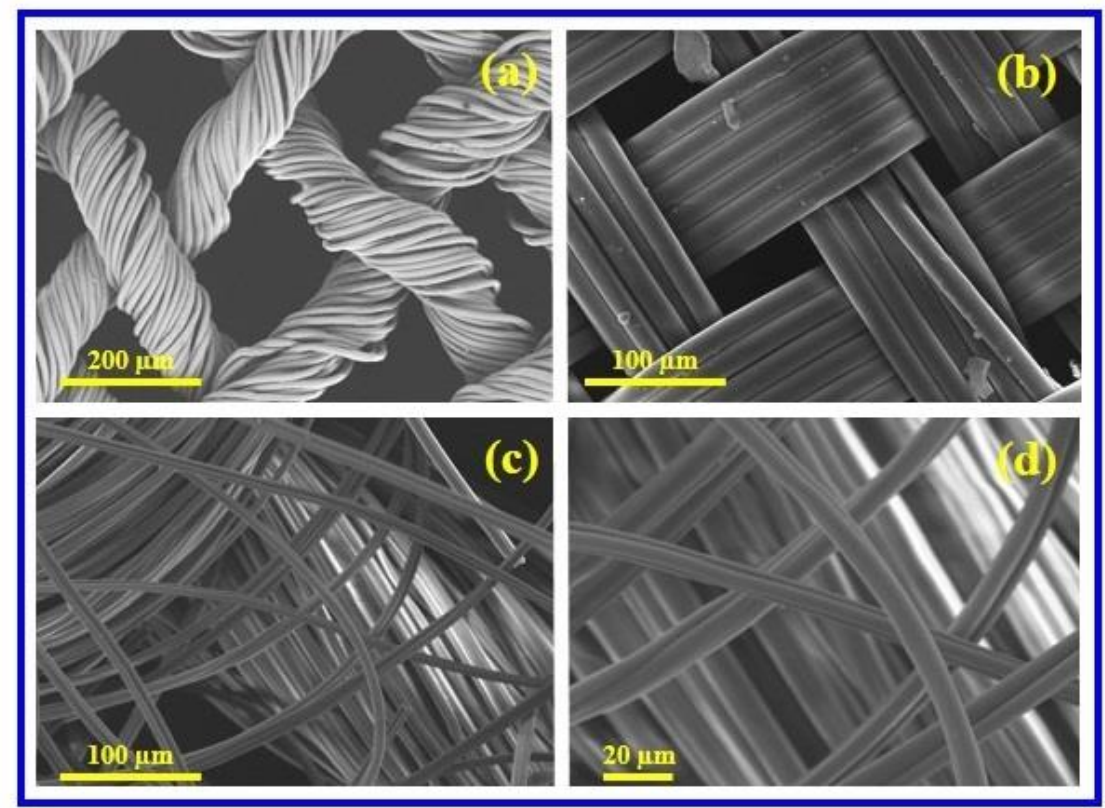

Figure 3. Enlarged images of fabric fibres: (a) Polyester; (b) Nylon, and (c) \& (d) Silk

A visual absorption study was carried out using the first treatment procedure. The procedure investigated the behaviour of various fabric samples towards the drop-wise application of $\mathrm{Au}$ nanoparticle solutions. The natural fabrics composed of silk, wool and cotton were investigated first. Silk tended to produce rapid solution spreading with little absorption. Unlike silk, wool displayed no solution spreading and absorption of the solution only occurred after a long contact period. While cotton exhibited no solution spreading, displayed very poor absorption and tended to repellent the test solution. The synthetics fabrics included nylon and polyester, with nylon displaying very good spreading ability and some absorption capacity. The polyester fabric tended to repel the test solutions and displayed poor spreading characteristics. Also examined were several commonly available fabric blends. The first was a wool-polyester blend (6W4P) that displayed good solution spreading and exhibited some repellence 
towards the test solution. Two wool-viscose blends were also examined. The first was a 50/50 blend $(5 \mathrm{~W} 5 \mathrm{~V})$ that displayed some surface repellence and moderate solution spreading. The blend (1W9V) immediately displayed a colour change when it came into contact with the test solution. The original fabric colour of grey changed to red and also displayed good solution spreading. The cotton-polyester blend (6C4P) permitted the test solution to absorb directly into the fabric and displayed poor solution spreading.

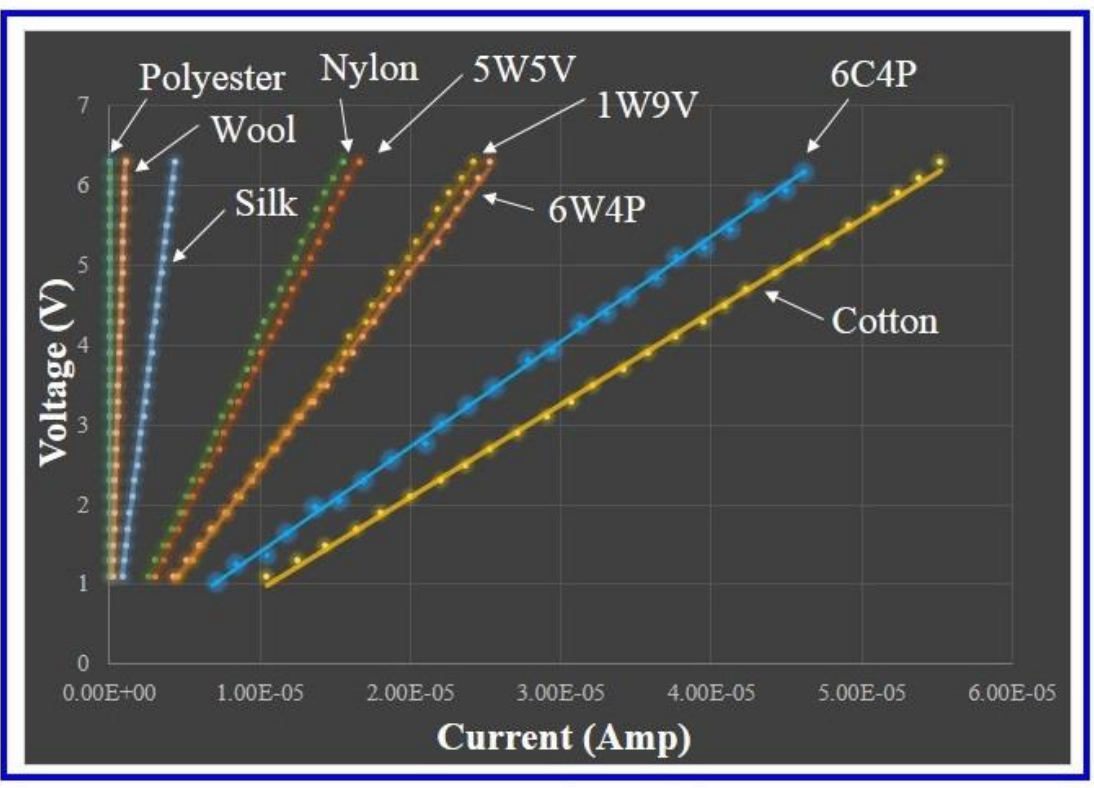

Figure 4. Voltage-current measurements for several fabric materials after a $73 \mathrm{~h}$ immersion treatment in an Au nanoparticle solution

Many natural and synthetic fibres used in textile fabrics have extremely high resistance values and their resistivity's are in the order of $10^{15} \Omega / \mathrm{cm}^{2}$. These extremely high resistance values equates to these fabrics been considered as insulating materials. In the present work, fabric samples were immersed in $\mathrm{Au}$ nanoparticle doping solutions for 20 and $73 \mathrm{~h}$ periods. After the immersion period, the samples were removed from the solution and allowed to air dry at room temperature $\left(24{ }^{\circ} \mathrm{C}\right)$. All pre-treated fabrics tested in the present work displayed extremely high resistances and could be considered as electrical insulators. After solution treatment all fabric samples underwent voltage-current measurements. These measurements revealed a linear relation that obeyed Ohm's law. A representative set of voltage-current trend lines for treated fabrics are presented in Figure 4 and reveal the highly linear nature of the measurements. Two immersion periods $(20 \& 73 \mathrm{~h})$ were investigated and both sets of voltage-current measurements gave similar results to those presented in Figure 4. The data was also analysed and the respective trend lines and $\mathrm{R}^{2}$ values were determined. The trend lines and $\mathrm{R}^{2}$ values for the $73 \mathrm{~h}$ treatment period are presented in Table 2. Inspection of both Figure 4 and Table 2 confirms the linear relationship of the voltage-current measurements and also confirms the measurements are indicative of Ohm's law. The post treatment results indicate polyester, wool and silk were still highly resistive. But, there was a noticeably reduction in their resistance values compared to pre-treatment measurements. In the case of cotton and other fabric blends there was a significant reduction in the resistance after solution treatment as seen in Figure 4. 
Table 2. Trend lines for voltage-current relationships for fabric materials examined in the present work.

\begin{tabular}{llll}
\hline Material type & $\begin{array}{l}\text { Sample } \\
\text { designation }\end{array}$ & Trend line & $\mathbf{R}^{\mathbf{2}}$ \\
\hline Natural & Cotton & $\mathrm{Y}=116041 \mathrm{x}-0.2151$ & 0.9991 \\
& Silk & $\mathrm{Y}=1 \mathrm{e}^{6} \mathrm{x}-0.1499$ & 0.9990 \\
& Wool & $\mathrm{Y}=6 \mathrm{e}^{6} \mathrm{x}+0.0136$ & 0.9998 \\
Synthetic & Polyester & $\mathrm{Y}=2 \mathrm{e}^{8} \mathrm{x}-0.1995$ & 0.9991 \\
& Nylon & $\mathrm{Y}=409228 \mathrm{x}+0.0162$ & 0.9996 \\
Blends & 6C4P & $\mathrm{Y}=223409 \mathrm{x}+0.2031$ & 0.9997 \\
& 6W4P & $\mathrm{Y}=246330 \mathrm{x}-0.0059$ & 0.9993 \\
& 5W5V & $\mathrm{Y}=387244 \mathrm{x}-0.0620$ & 0.9997 \\
& 1W9V & $\mathrm{Y}=266887 \mathrm{x}-0.1827$ & 0.9990 \\
\hline
\end{tabular}

Note: $\mathrm{Y}$ is the applied voltage and $\mathrm{x}$ is the measured current flow in amps

The final set of voltage-current measurements was designed to test the feasibility of using a treated fabric (silk) to detect the presence of ethanol vapour via a change in material electrical resistance. Voltage-current measurements were carried out and the results presented graphically in Figure 5. Testing revealed treated silk fabrics responded to the presence of ethanol vapour. Prior to exposure to ethanol vapour, silks voltage-current trend line (yellow) was fair steep, but after exposure the slope (green) was appreciably reduced indicating a significant decrease in resistance. Thus, solution treated silk fabrics can be used to detect the presence of ethanol vapour and in principle could be used as potential wearable chemical sensor. However, further follow up studies are needed to fully examine the behaviour of the various fabric materials and to optimise their respective resistive properties under similar nanoparticle based treatments. The present work has highlighted the potential of incorporating current commercially available fabric materials into the manufacture of wearable chemical sensors.

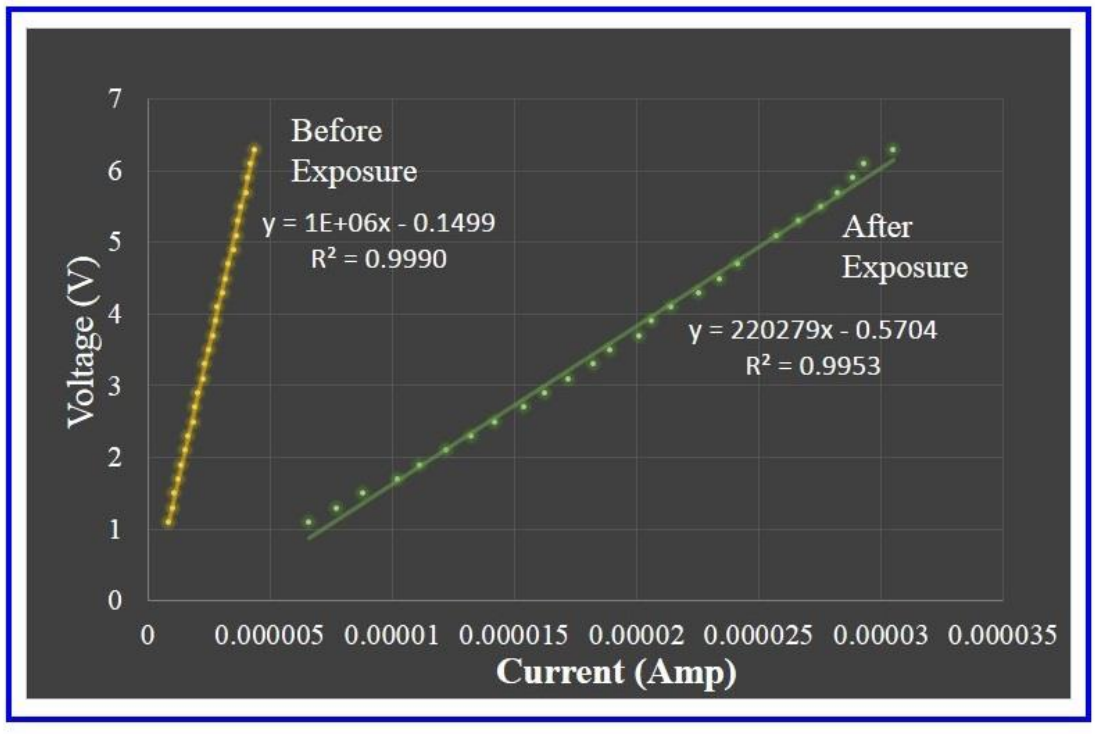

Figure 5. Voltage-current measurements carried out on a treated silk fabric before and after exposure to ethanol vapour.

\section{Conclusion}

The present work has determined the voltage-current characteristics of several natural, synthetic and natural/synthetic blend fabrics before and after treatment with $\mathrm{Au}$ nanoparticle solutions. The data was derived from a two probe voltage-current method. Analysis of the results has confirmed that all treated fabrics obey Ohm's law and have the potential to be incorporated into wearable sensors. The study also revealed that a treated silk fabric could be used as a chemical sensor to detect the presence of ethanol vapour. However, further research is needed to fully examine the physiochemical nature of the treated fabric materials, durability and their respective responses to a wider range of chemicals. The future work will also involve developing prototype devices specifically designed to sense the presence of particular chemical materials. 


\section{Acknowledgements}

Derek Fawcett would like to thank Horticulture Innovation Australia for their research support through Project Al14003.

\section{References}

1) Wilson, S.A., Jourdain, R.P.J., Zhang, Q., et al. 2007. New materials for micro-scale sensors and actuators: An engineering review. Materials Science and Engineering R. 56: $1-129$.

2) Wales, D.J., Grand, J., Ting, V.P., et al. 2015. Gas sensing using porous materials for automotive applications. Chem. Soc. Rev. 44: 4290-4321.

3) Bhandari, B., Lee, G.Y., Ahn, S.H. 2012. A review on IPMC material as actuators and sensors: Fabrication, characteristics and applications. International Journal of Precision Engineering Manufacturing. 13(1): 141-163.

4) Ragazzo-Sanchez, J.A., Chalier, P., Chevalier-Lucia, D., et al. 2009. Off-Flavours detection in alcoholic beverages by electronic nose coupled to GC. Sens. Actuators B. 140: 2934.

5) Barbri, N.E., Mirhisse, J., Ionescu, R., et al. 2009. An electronic nose system based on a micro-machined gas sensor array to assess the freshness of sardines. Sens. Actuators B. 141: 538-543.

6) Zappa, D., Comini, E., Zamani, R., et al. 2013. Preparation of copper oxide nanowire-based conductometric chemical sensors. Sensors and Actuators B: Chemical. 182: 7-15.

7) Hoa, N. D., Quy, N.V., Jung, H., et al. 2010. Synthesis of porous $\mathrm{CuO}$ nanowires and its application to hydrogen detection. Sensors and Actuators B: Chemical. 146(1): 266272.

8) Branca, A., Simonian, P., Ferrante, M., et al. 2003. Electronic nose based discrimination of a perfumery compound in a fragrance. Sens. Actuators B. 92: 222-227.

9) Windmiller, J.R. and Wang, J. 2013. Wearable electrochemical sensors and biosensors: a review. Electroanalysis. 25: 29-46.
10) Turner, A. 2013. Biosensors: then and now. Trends Biotechnol. 31: 119-120.

11) Bandodkar A.J., Joseph Wang, J. 2014. Non-invasive wearable electrochemical sensors: a review. Trends in Biotechnology. 32(7): 363-371.

12) Patel, S., Park, H., Bonato, P., et al. 2012. A review of wearable sensors and systems with application in rehabilitation. Journal of NeuroEngineering and Rehabilitation. 9(21): 1-17.

13) Bonato, P. 2010. Wearable sensors and systems. From enabling technology to clinical applications. IEEE Eng. Med. Biol. Mag. 29: 25-36.

14) Guinovart, T., Parrilla, M., Crespo, G.A., et al. 2013 Potentiometric sensors using cotton yarns, carbon nanotubes and polymeric membranes. Analyst. 138(18): 5208-5215.

15) Chinta, S.K., Gujar, P.D. 2013. Significance of moisture management for high performance textile fabrics. International Journal of Innovative Research in Science, Engineering and Technology. 2(3): 814-819.

16) Schazmann, B., Morris, D., Slater, C., et al. 2010. A wearable electrochemical sensor for the real-time measurement of sweat sodium concentration. Anal. Methods 2, 342-348.

17) Jia, W., Bandodkar, A.J., Valdes-Ramirez, G., et al. 2013 Electrochemical tattoo biosensors for real-time noninvasive lactate monitoring in human perspiration. Anal. Chem. 85, 6553-6560.

18) Patel, S., Hughes, R., Hester, T., et al. 2010. A novel approach to monitor rehabilitation outcomes in stroke survivors using wearable technology. Proceedings of the IEEE. 98: 450-461.

19) Adomaitiene, A., Kumpikaite, E. 2011. Analysis of mechanical properties of fabrics of different raw materials. Materials Science (Medziagotyra). 17(2): 168-173.

20) Hersh, S.P., Montgomery, D.J. 1952. Electrical resistance measurements on fibres and fibre assemblies. Textile Research Journal. 22(12): 805-818. 